\title{
Conscience du sentiment - conscience de sentiments. Le sentiment comme acte et comme état
}

Texte V (§ 4-6) in Edmund Husserl, Études concernant la structure de la conscience (1908-1914), volume 2, « Actes affectifs et donnée axiologique »

\section{Edmund Husserl}

Traducteur : Claudia Serban, Natalie Depraz et Maria Gyemant

\section{OpenEdition} Journals

Édition électronique

URL : http://journals.openedition.org/alter/437

DOI : $10.4000 /$ alter.437

ISSN : 2558-7927

Éditeur :

Association ALTER, Archives Husserl (CNRS-UMR 8547)

\section{Édition imprimée}

Date de publication : 1 décembre 2016

Pagination : 231-248

ISBN : 978-2-9550449-2-6

ISSN : $1249-8947$

Référence électronique

Edmund Husserl, «Conscience du sentiment - conscience de sentiments. Le sentiment comme acte et comme état», Alter [En ligne], 24 | 2016, mis en ligne le 01 décembre 2017, consulté le 30 avril 2019. URL : http://journals.openedition.org/alter/437 ; DOI : 10.4000/alter.437 
$<$ conscience du sentiment - conscience de sentiments. Le sentiment comme acte et comme état>

$<\S 4$. Le plaisir comme acte et l'affect de la joie comme état $>$

[A VI 12 II/126 a "46"] La joie comme acte qui vise, donc aussi comme conversion attentionnelle (Zuwendung) (plaisir-pris-à, seréjouir-de).

I a) Vivre dans l'acte et trouver le caractère de la splendeur à même l'objet.

II) La joie comme état, vivre dans l'état de la joie, sans se tourner vers ce dont on se réjouit, sans rapporter à cela l'état de joie.

II a) On peut enfin vivre dans l'état de la joie et regarder dans la direction de l'objet et se dire qu'il est ce dont on se réjouit, qu'il est ce qui nous dispose à la joie, qui nous rend gais.

$<$ ad> I) Donc d'abord l'acte. Ne devrions-nous pas dire que l'acte, $c^{\prime}$ est l'agrément pris à quelque chose (Wohlgefallen an) ? J'entends une nouvelle réjouissante, je l'entends avec un sentiment de bonheur, je l'entends avec beaucoup de satisfaction (Wohlgefallen), avec grande joie, avec des sentiments de bonheur.

$<\mathrm{ad}>$ II a) Je dis au messager: Je m'en réjouis de tout mon cœur. Cela me rend heureux (cela me remplit de bonheur, de joie).

$<\mathrm{ad}>\mathrm{I}$ a) Je peux aussi dire : Cela est beau, c'est merveilleux. Mais j'ai ici une autre appréhension [de la chose]. L'objet a ici, pour moi, le caractère de la "beauté ", un caractère axiologique. C'est cela que j'exprime. Je suis tourné vers (zugewendet) la nouvelle [qui vient de m'être annoncée] et ce, non seulement sur le mode de la représentation, mais sur le mode du sentiment. La conversion attentionnelle du sentiment est un plaisir joyeux. Cela ressemble à la manière dont, dans la contemplation d'une image (et non pas simplement de l'objet 
que l'image représente), je peux avoir un plaisir ou un déplaisir esthétique. Que ceci ou cela advient ou est advenu, je le vois ou l'entends avec joie, avec la conversion attentionnelle de la joie. Je me plais à regarder un spectacle de gymnastique, ces monstrations de la force des hommes, de l'aisance, de l'audace. Il s'agit là, visiblement, non seulement de conversions attentionnelles, mais de [visées] thématiques. Je vis thématiquement dans le sentiment. Une odeur agréable, que m'apporte un courant d'air, peut me toucher sur le moment et obtenir mon attention (Zuwendung), mais accessoirement.

[126 b] Qu'en est-il maintenant de l'état de la joie? L'acte de la joie se tournant vers quelque chose, de la visée de la joie, se transforme dans l'état de la joie, l'état du bonheur, de la félicité, dans la « disposition » durable, dans l'affect de la joie qui peut éventuellement être très mouvant et changeant quant à son contenu. Quelle sorte de différence avons-nous à saisir ici ? S'agit-il d'une différence qui concerne toutes les sphères de la conscience ? On pourrait rassembler ensuite quelques exemples: le doute comme acte, le doute comme état (et non plus comme accomplissement actuel de "est-ce A ou non ? » et ainsi de suite), le jugement comme acte: "S est $\mathrm{P}$ », le jugement comme état de croyance. Le décider comme acte, l'état de la décision; le souhaiter comme acte, le souhait comme état (l'état désirant); le manque douloureux comme acte et état de la privation. Examinons de plus près le cas de la joie!

Je regarde le spectacle de gymnastique avec satisfaction. Lorsqu'il est terminé, je l'avais regardé avec satisfaction, l'«acte» est là-dedans, de même que la perception et les jugements de perception sont là-dedans. J'entends la bonne nouvelle avec satisfaction. Après $<\mathrm{l}^{\prime}>$ avoir entendue, la satisfaction qu'elle m'a donnée y est [présente] comme acte. De même que l'acte de jugement. Je m'en réjouis, mais « encore ». De même que je crois encore que j'ai raison à propos de cela ou cela, etc. ? Maintenant, une chose est certaine : l'acte est certes passé, mais il est bien éprouvé en tant qu'il s'installe et s'écoule, accompagné d'une modification. Il ne s'agit pas de la simple modification d'un souvenir frais. Car j'ai « encore » la satisfaction, je juge " encore », je me tiens encore sur le terrain du plaisir et sur le terrain du jugement. Si j'accomplis un ressouvenir, si je récapitule pour ainsi dire, je confirme la prise de position, je ne la supprime pas. Non seulement cela m'a plu, mais cela était beau ; non seulement j'ai ressenti de la satisfaction, mais c'était quelque chose de satisfaisant. Et la chose que j'ai partagée, qui continue d'agir et perdure est toujours et encore réjouissante. Nous avons donc plus qu'un souvenir de la prise de position, plus qu'un ressouvenir ou un souvenir rétentionnel : la 
rétention est aussi rétention de la prise de position elle-même, elle est maintenue comme prise de position.

[A VI 12 II/127 a "47"] Par conséquent, la conscience d'acte - la conversion attentionnelle actuelle et la visée - perd certes le caractère de l'actualité dans le sens spécifique de commencement et d'écoulement originaires, mais elle se maintient dans une modification dans laquelle la prise de position perdure sous la forme d'un état et reste donc retenue et maintenue. Parallèlement se produit un obscurcissement. La représentation, le jugement et les actes éventuellement édifiés sur eux s'obscurcissent - en quel sens ? En effet, les représentations claires, actuelles et évidentes se changent en rétentions pâles, en représentation $<\mathrm{s}>$ confuses et vides. De même pour les jugements : ils deviennent des copies vides. L'orientation vers des objets, états de choses, valeurs et états de valeur est là, mais sans « clarté et évidence (Deutlichkeit)».

Mais cela n'épuise pas la chose. Quand la nouvelle réjouissante est entendue, quand $\mathrm{l}^{\prime}$ [acte de $\mathrm{l}^{\prime}$ ]entendre <est> écoulé, je suis encore dirigé vers elle en tant que bonne nouvelle dans un agrément rétentionnel de la joie - fût-ce dans une conscience vide - ; la nouvelle est toujours et encore mon thème, ce vers quoi je tends pour ainsi dire. De même dans le cas d'un jugement : je lis l'énoncé avec conviction, et après l'avoir lu je suis encore dirigé vers l'état de choses dans un état de croyance. Je le vise encore. L'évidence de l'être-dirigé est assurée, même s'il n'en est pas de même pour la clarté. Mais l'orientation primaire peut devenir secondaire, je peux me tourner vers un nouveau jugement tout en tenant encore l'ancien, etc. Nous avons déjà parlé de cela.

Mais comment cela se passe-t-il pour la joie? Je demeure dans l'état de joie sans rester à proprement parler, pendant cet état, continuellement dirigé vers ce qui est réjouissant, et tout comme dans le cas du jugement à l'intérieur d'un thème englobant de nouvelles connexions se forment, quelques conséquences réjouissantes peuvent s'ensuivre et se maintenir continuellement. Dans tous les cas, même lorsque le rapport à ce qui est réjouissant perdure d'une certaine manière pendant l'état de la joie, il semble pourtant que l'orientation (Richtung) vers l'objet se passe tout autrement que dans le cas du jugement. En effet, il y a ici quelque chose de nouveau qui est à prendre en compte.

L'analogon, et même l'analogon complet du jugement, est la satisfaction. Nous distinguons cependant la satisfaction de la joie qui me fait frissonner, etc. La satisfaction, l'acte, ne me fait pas frissonner, mais suscite le frisson de la félicité. [A VI 12 II/128 a "48"] La satis- 
faction, l'acte au sens véritable, la conversion attentionnelle et la "visée », est la source d'un affect de la joie, la source d'une gaieté, $d^{\prime}$ 'une humeur joyeuse, d'une disposition joyeuse. On peut presque dire que dans la plupart des cas le mot «joie» désigne l'affect, la disposition et non pas l'acte. (Le mot « affect » renvoie à l'excitation, au fait d'être tiré vers le haut, vers les «crêtes» d'un sentiment orageux ou vers ses vallées profondes, tandis que le mot « disposition » renvoie plutôt à un niveau uniforme, à la vivacité égale et constante du sentiment à un niveau positif, ou à la disposition basse à un niveau plus profond.) Au lieu de dire: "cela me plaît », nous disons en effet: «j' éprouve de la joie à ce propos ». Ici, l'acte ressortit davantage. "Cela me remplit de joie»: cela renvoie davantage à l'affect.

De nombreux exemples peuvent nous aider à comprendre $\mathrm{qu}^{\prime}<\mathrm{une}>$ telle différence est à faire et qu'effectivement l'acte de plaisir (Gefallensakt) est à regarder comme source d'un affect. Je vois la gymnastique et cela me plaît, la progression de la représentation éveille une joie toujours plus vive, j'en viens à une excitation joyeuse toujours croissante, éventuellement à une vraie tempête de ravissement. (Au lieu de la gymnastique, cela pourrait aussi être un jeu de pari sur des ballons, une régate, etc., et le spectateur qui est réceptif à cela. Dans le cas du pari, je prends parti et je m'y intéresse avec mes envies, mes engagements, etc. : il vaut cependant mieux exclure tout cela au début.) Je passe d'actes de l'agrément à de nouveaux et encore de nouveaux [actes]. Ce qui vient tout juste de se produire me plaît autrement que s'il était arrivé au début de la présentation. Cela ne plaît pas en tant que meilleur. Je lui attribue peut-être une moindre valeur ou je l'estime digne d'une moindre valeur, lorsque je compare. Et pourtant, mon sentiment de bien-être s'accroît, j'atteins une plus grande excitation, la joie devient de plus en plus grande, je m'installe progressivement dans la disposition affective (donc affect et disposition affective).

[128 b] Maintenant se pose la question de savoir comment cela peut être explicité. L'acte et l'affect doivent-il être séparés? Ou bien avons-nous à distinguer, dans la même conscience de plaisir, deux " côtés ": la satisfaction comme attitude axiologique et une composante qui connaît l'accroissement, la composante de la joie et de l'excitation joyeuse, qui s'accroît (généralement) en fonction de la durée et du nombre d'actes d'agrément? D'un côté, l'attribution de valeur, $\mathrm{d}^{\prime}$ un autre, la jouissance ; la dernière [correspond] au plaisir comme état, <pensé> comme passivité, la première, à la prise de position propre à l'attribution de valeur, comme activité. Mais l'activité et la 
passivité sont-elles les deux côtés d'une seule et même chose? Et l'on pourrait objecter :

1) Deux «côtés »? Ne pouvons-nous pas « rester froids », maussades, incapables de jouir? Ne se peut-il pas que nous ayons de l'appréciation, et en ce sens du plaisir, sans être le moins du monde excités, sans nous réjouir, sans jouir ? Ainsi, par exemple, dans le cas de l'attribution d'une valeur esthétique : fatigué par le voyage, je me rends à la galerie de peinture, je vois beaucoup de belles choses, des œuvres d'art sublimes et pleines d'attrait, j'appréhende leur beauté, je la contemple esthétiquement. Mais la «jouissance» véritable est réduite, je n'atteins pas un état de ravissement heureux, un courant de joie, comme lorsque je suis frais corporellement et spirituellement. Il y a certes une sorte d'excitation, mais elle n'arrive pas à se développer: la fatigue et sa disposition affective (Gefühlsstimmung) prédominent et retiennent le reste. Il en est de même avec les sentiments eu égard aux personnes de mon entourage dans les rapports sociaux. Je parle avec un nouveau collègue. Chacune de ses manifestations me plaît, sa personnalité tout entière se tient devant moi dans un agrément (Wohlgefallen) constant. Et pourtant je ne deviens pas chaleureux. Une autre fois je deviens gai, un courant d'allégresse et de chaleur me traverse, etc.

Toutefois la portée de cet argument est problématique. L'on pourrait dire que c'est la chose qui fait que chaque plaisir, selon ses composantes d'état, a son degré de chaleur, que parfois lorsque le plaisir succède au plaisir, le plaisir passé est encore « agissant » et que cette efficace ultérieure accroît le degré de chaleur des nouveaux actes de plaisir. Et pourtant l'expression « degré de chaleur» peut égarer. [A VI 12 II/129 a "49"] Mieux vaut parler de degré d'excitation ou de température. Une nouvelle fâcheuse ou, mieux, une odeur désagréable sont déplaisantes et ont leur degré. Lorsque d'autres mauvaises odeurs me gênent encore et encore, le degré d'excitation de chaque nouveau sentiment s'accroît. Dans l'exemple ci-dessus, je reste froid, cela voudrait dire: la température des sentiments se maintient à un niveau inférieur. Mais le plaisir est et demeure plaisir, et le déplaisir déplaisir. Dans le premier cas, la composante de la jouissance ou de la souffrance (du plaisir et du déplaisir véritable) connaît un accroissement, dans l'autre cas, non. Selon l'autre conception, l'on dirait que la température peut aussi être égale à zéro et qu'il ne s'agit pas de côtés, mais seulement d'une composante associée.

2) Or il faut à présent prendre en compte la différence entre la température du « côté » ou de la composante "état»(Zustand), et celle de la disposition (Stimmung) persistante et unitaire de part en 
part. Cela nous apprendra peut-être quelque chose de particulier, tout comme l'état d'excitation qui persiste ou l'affect. D'un côté, nous avons l'unité de l'acte ou la pluralité d'actes qui se suivent et entre lesquels il n'y a pas de cohésion (par des actes de liaison): je vais dans la galerie, dans l'exposition artistique, de tableau en tableau, j'éprouve de la satisfaction ou encore du plaisir. D'un autre côté, les excitations de plaisir et de sentiment se nouent dans l'unité d'une disposition.

Plus loin encore : même lorsque tous les actes y sont, une disposition élevée et prégnante se maintient, un affect positif ou négatif. Les actes continuent d'agir, mais je ne les accomplis plus, je ne les retiens pas non plus, comme il advient pour les actes sur lesquels, pour autant qu'ils perdurent dans la rétention (dans la mesure où je les maintiens ensemble avec leur prise de position), de nouveaux actes $<$ se $>$ fondent.

Il faut aussi rappeler ici la chose suivante : Je parle à une personne chère, je suis tourné vers elle dans l'agrément et j'atteins l'état d'une excitation chaude, éventuellement heureuse. Je peux aller plus loin maintenant et, tourné vers elle dans le souvenir, continuer de vivre encore l'agrément et la joie. [129 b] Mais même lorsque je me tourne vers d'autres objets, la « disposition » heureuse et l'excitation d'abord joyeuse qui avait diminué progressivement demeurent. De même pour la disposition élevée après la « jouissance » d'un chef-d'œuvre. Ici encore, il faut distinguer d'une part l'agrément et la jouissance dans la conversion attentionnelle, et d'autre part la disposition ou l'excitation qui persistent par la suite.

En allant encore plus loin, on peut considérer : Je vois une œuvre $d^{\prime}$ art et je lui attribue de la valeur en tant qu'œuvre d'art, autrement dit, je "saisis sa beauté ", non pas toutefois sur le mode de la représentation, mais la beauté s'impose à moi dans l'attribution de valeur, dans la « reconnaissance » esthétique. Je tombe à présent dans un état de félicité, je jouis de l'œuvre, je m'en réjouis. Je suis traversé par le frisson du plaisir esthétique. Une personnalité noble se tient devant moi, je "sens sa valeur» dans une conscience de valeur. Je tombe dans une allégresse, un sentiment de bien-être me traverse, et c'est le même qui se maintient encore lorsque l'attribution de valeur est terminée. Je ressens ma joie «à même » la personne. L'œuvre d'art me procure de la joie, j' en jouis. Est-ce que je jouis de la chose et est-ce cela qui suscite ma joie, me rend-elle joyeux en tant que chose? Ou bien la personne noble: est-ce son existence, est-ce la personne existante qui éveille la félicité ? Je peux naturellement adopter aussi cette attitude causale et parler ainsi. Mais il ne s'agit pas de cela ici. Il 
vaut mieux dire déjà ceci : la beauté de l'œuvre d'art éveille et suscite ma joie et, " adonné » à ce plaisir (c'est-à-dire, sans l'observer d'une quelconque manière, sans l'objectiver dans un fait psychologique), je jouis ${ }^{1}$. Plus loin encore, je peux dire : cette jouissance a sa source dans le beau, dans la conscience de la beauté ; elle passe par la suite dans la disposition élevée et gaie qui a sa source quant à elle, non pas dans le beau, ou plutôt, non pas dans la conscience de la beauté, dans la conversion attentionnelle vers elle, dans sa saisie et dans la manière dont la conscience la possède, mais dans la jouissance?

[A VI $12 \mathrm{II} / 71 \mathrm{a}$ " 50 " "Ax"] Le plaisir pris au beau, la jouissance, est un foyer de rayonnement, la source d'une disposition, il est éventuellement excitation, en tout cas " plaisir », et à partir de lui émane une nouvelle excitation. Nous faisons la même distinction également dans le cas de la jouissance sensible élémentaire : je mange le strudel ; ce que j'ai dans la bouche a un bon goût. Je jouis, et de cela émane un sentiment de bien-être comme une disposition agréable et joviale, qui peut éventuellement se maintenir longtemps. Dans d'autres cas, cela peut se passer ainsi : le strudel est bon, mais je ne peux pas en jouir adéquatement, la jouissance ne me procure aucune joie, elle ne se propage pas, aucune disposition joviale (joie du ventre) ne se répand à partir d'elle.

$<\S 5$. Plaisir sensible, jouissance, disposition et sentiments intentionnels de valeur $>$

La disposition est motivée. Quand je me trouve bien disposé, quand je chante de joie, etc., je vis du plaisir et même, éventuellement, je prends plaisir au plaisir dans des « actes » divers. «Tout me paraît beau maintenant. » Je deviens aussi réceptif aux beautés effectives, je me tourne vers elles. Je chante une belle chanson et je m'en réjouis. La même chanson ne $\mathrm{m}^{\prime}$ apportait pas tant de jouissance autrefois, mais maintenant le degré de la chaleur du plaisir propre à cette jouissance est grand. Tout prend couleur, tout est réchauffé à partir de la disposition, tout plaisir est intensifié, reçoit un afflux de chaleur qui n'a pas sa source dans l'objet de valeur, et ce qui est indifférent devient presque quelque chose de «beau» (à savoir, il reçoit une brillance d'emprunt). Je le sais aussi. Je peux dire : «je suis heureux, gai, joyeux, parce que j'ai pris un repas tellement bon, parce

\footnotetext{
${ }^{1} \mathrm{Ou}$ bien est-il plus juste de dire que la beauté est le corrélat du sentiment de joie esthétique, et que cette joie est alors la source d'affects qui rayonnent, de sentiments?
} 
que j'ai joui d'œuvres d'art tellement belles, parce que j'ai remporté un succès (j'en ai reçu la nouvelle), et ainsi de suite. Tout me procure une double joie, parce que...». Motivé ne veut pourtant pas dire justifié, mais exprime seulement le rayonnement, et le fait que je trouve dans le phénomène lui-même que le plaisir n'a pas sa source dans la valeur de l'objet lui-même, mais renvoie en arrière à une attribution de valeur et une jouissance antérieures comme en "provenant». Ces réflexions du sentiment (Gefühlsreflexe) (la brillance d'emprunt) ne fondent aucune valeur, même pas des valeurs dérivées ([celles-ci seraient] plutôt sources d'erreurs axiologiques).

[71 b] Lorsque nous tentons d'isoler la conscience dans laquelle la chose est consciente en tant que porteuse d'une valeur, et le plaisir comme état, comme jouissance, nous parlons du côté de la jouissance de degrés d'accroissement, ou encore de propagation, de rayonnement, et ces derniers peuvent se délier des estimations axiologiques actuelles. Si nous maintenons ce lien, nous remarquons des différences comme : je regarde le tableau avec plaisir, un frisson de bonheur «me» traverse. $\underline{\mathrm{Me}}$ moi : à travers mon corps passe un courant de plaisir, je sens ce bonheur dans le cœur, dans la poitrine, les frissons vont jusqu'aux orteils, etc. L'on pourrait pourtant dire que ce ne sont que des sentiments purement sensibles. En tant que reliés à des parties du corps, ils ne sont pas ce au sujet de quoi il y a joie, ils ne sont pas l'objet du plaisir. Je ne prends pas plaisir, je ne ressens pas de la joie au sujet de mon cœur, de ma poitrine. Cela ne reçoit pas de valeur, ne se trouve pas là en tant que beau, sa bonté et beauté ne suscitent aucun plaisir, mais je me réjouis au sujet de l'œuvre d'art, au sujet de la vision (saisie axiologique) du beau, je me réjouis au sujet de la bonté de cet homme (qui est consciente comme caractère de valeur du beau).

La liaison entre l'état de joie, le frisson de bonheur, etc., et l'appréhension du corps propre et des parties de ce corps, est essentiellement différente de la liaison entre le plaisir pris à la nourriture et l'appréhension de la nourriture. La nourriture a un bon goût, procure un plaisir ; ma poitrine n'est " pas bonne » et ne me procure pas un plaisir au même sens. D'autre part, l'on pourrait dire : c'est le sentiment de bien-être, le doux plaisir (la douleur tracassante) dans la poitrine, mais non pas qu'il s'agit là d'un objet déterminé comme objet du plaisir. Bien plutôt, <il y a là > une teneur de sensation entrelacée avec du plaisir, tout comme dans le cas des sensations épidermiques, où nous ne disons pas: je me réjouis de ma peau, ma peau me procure du plaisir, etc. Ne doit-on pas dire pourtant qu'il s'agit 
de plaisirs sensibles, qui ne font que rayonner à partir du plaisir d'origine?

[A VI 12 II/74 b "51" “Ax"] Et qu'en est-il alors des dispositions qui s'ensuivent? Doit-on dire qu'elles sont en soi des sentiments sensibles ? Je suis "mal disposé ", dans une disposition oppressante. Je suis bien disposé, dans une disposition élevée. Peut-on dire : d'un côté, il s'agit ici à coup sûr d'un contenu de sensation, et celui-ci <est> porteur de sentiments sensibles agréables ou désagréables confondus dans une vague unité ? D'un autre côté, il s'agit de sentiments qui ont bien leur source: je suis oppressé car j'ai traversé une épreuve difficile. Cela se trouve à l'arrière-plan. Mais je n'y pense pas, cela ne se trouve pas effectivement (pas généralement) à l'arrière-plan comme représentation du malheur vécu. Ou bien je suis dans une disposition élevée, j'ai vu quelque chose de merveilleux, j'ai été tout à l'heure à Pitti, j'ai vécu brièvement une grande joie. Je ne pense pas à tout cela maintenant, cela n'est pas effectivement représenté à l'arrière-plan de la conscience. Mais cela continue d'agir, et la disposition joyeuse ou élevée y renvoie encore ${ }^{2}$.

Ce caractère originel différencie les dispositions qui, en soi, selon leur teneur de sentiment, selon leur écoulement et leur forme d'unité, ne se laisseraient guère différencier. La félicité qui me traverse à la manière d'un frisson doux peut tout aussi bien être exactement la même à la vue d'un grand chef-d'œuvre de la peinture, à l'occasion d'une symphonie, mais aussi à la vue de la personne aimée, de la femme qui s'abandonne, etc., et éventuellement aussi dans le cas de la félicité religieuse. Il s'agit d'annexes de sentiments sensibles, d'extensions, de propagations, de rayonnements de sentiments originels, caractérisés ainsi à chaque fois et déterminés quant à leur valeur seulement à partir de cette cohésion.

[A VI 12 II/74 a] L'on pourrait ajouter que la manière dont les annexes sensibles jouent leur rôle est significative d'un point de vue éthique : l'homme pieux qui d'une certaine manière s'élève au frisson de la félicité peut devenir quelqu'un qui jouit de la religiosité. Au lieu de vivre dans des sentiments axiologiques religieux, de s'adonner à une valeur du divin, il vit dans la jouissance du frisson sensible, qu'il sait aussi susciter d'une manière impropre à travers des cérémonies qui ont perdu leur signification, des formes ecclésiastiques, etc. Et ne doit-on pas dire quelque chose de semblable à propos de l'esthète? Il

\footnotetext{
${ }^{2}$ Mais ne s'agit-il pas là d'actes obscurs d'attribution de valeur et de représentations du fond de la conscience? Non pas, certes, au sens d'aperceptions intuitives se trouvant en dehors de la sphère $\mathrm{d}^{\prime}$ attention.
} 
n'est pas tant adonné à la valeur du beau ou à la pure joie que ce dernier procure, qu'à la jouissance que cette joie apporte avec soi et propage, et dans ce cas des jouissances semblables peuvent surgir aussi à partir de sources douteuses esthétiquement.

Ne puis-je pas aussi distinguer l'acte et l'état? L'acte (la prise de position) est dirigé intentionnellement vers le bien, le beau, etc., il a une orientation (Hinwendung) vers eux et une visée. Lorsque je me réjouis $\mathrm{du}$ beau - d'une manière active, tourné vers lui, le contemplant ensemble avec les motifs de joie qui le traversent ${ }^{3}$, qui constituent la valeur -, j'ai aussi le vécu des rayonnements dans la forme de la joie sensible, des excitations, des affects (je suis affecté, et j'y suis portant passif). Je vis des sentiments de bien-être, un frisson sensible, etc. À cet égard je vis des situations qui ont le caractère d'un état. Je ne suis pas tourné vers le sensible, je n'accomplis à cet égard aucun acte (comme des conversions attentionnelles et des visées) ${ }^{4}$. Je peux pourtant me tourner vers lui, à savoir vers ce frisson, vers ce doux bien-être, et cela peut devenir à nouveau objet de la joie, d'une jouissance. On s'y habitue et on le prend pour thème. On s'installe dans le bien-être, ce qui l'intensifie, etc. Cela semble vraiment suggérer que les sentiments sensibles s'identifient à des sensations et ne sont pas des actes. Un acte serait ici le plaisir pris au doux frisson, comme le plaisir procuré par la saveur de la nourriture.

[A VI 12 II/130 a "52"] Je voudrais adopter la position suivante : 1) Lorsque je m'attriste de la perte de mon ami, il s'agit là d'un acte de non-joie à propos de cette perte ; quelque chose qui était là en tant que valeur, n'est plus, et le non-être du bien, le ne-plus-être, suscite la douleur. La non-joie dirigée contre ce non-être est l'acte de la tristesse. Lorsque je me réjouis de l'homme qui m'est cher et de sa façon d'être dans le discours et l'action, par exemple dans le dialogue avec lui, il s'agit là d'un acte qui se tourne vers lui sur le mode de la joie (Akt der Freudenzuwendung), de l'inclination amoureuse. Dans cet acte, j'ai conscience de lui et de sa façon d'être comme "chers », et son existence est réjouissante. C'est là le corrélat ontique de l'acte (qui peut devenir objectivement conscient dans la réflexion), et l'un et l'autre sont inséparables.

\footnotetext{
${ }^{3}$ Cette joie serait la joie originelle et identique au plaisir de l'attribution de valeur.

4 Tout cela est juste, mais ne prend pas en considération le fait que dans l'amour affectif, dans l'enthousiasme, etc., je suis tourné de manière amoureuse (liebend zugewendet), et que l'on doit dire que la propagation sensible, la quantité d'affection, reçoit elle-même une fonction intentionnelle. Conformément à cela, l'objet se tient là non pas seulement comme plaisant, mais comme splendide, etc.
} 
2) Comment la joie et la tristesse comme acte se rapportent-elles à l'état, à la disposition de la joie ou de la tristesse, et à l'affect de la joie, de l'allégresse, de la gaieté ? Pendant que je parle à l'homme qui $\mathrm{m}^{\prime}$ est cher, la joie peut déjà passer dans une excitation joyeuse, dans un affect. On peut tout aussi bien dire: dans une disposition joyeuse. Il s'agit tout d'abord dans notre exemple d'un enchaînement d'actes de joie qui ont une cohésion thématique. Chaque acte de cette sorte et l'unité de l'éventuel acte global est d'un côté un agrément dans lequel on a conscience d'un caractère de valeur et qui, pour autant qu'il y a en lui conscience d'une valeur, connaît des différenciations concernant le degré de la valeur, mais non pas concernant la vivacité. D'un autre côté, je peux en être joyeusement ému d'une façon plus ou moins profonde, ou tristement ému d'une façon plus ou moins profonde, [je peux] être triste plus ou moins profondément. Mon " intérêt » n'est jamais d'une profondeur égale, je suis intéressé, je participe avec mon ego, tantôt superficiellement, tantôt plus profondément.

Ces degrés de la participation de l'ego, de l'être-intéressé, de l'immersion du sentiment dans mon ego et de mon ego dans le sentiment, sont sans doute quelque chose de particulier qui ne s'identifie pas sans reste avec l'excitation plus ou moins grande. Je peux être très profondément affecté, très profondément ému, la joie peut être très profonde sans qu'elle montre le caractère d'une grande excitation, sans qu'elle évolue dans une allégresse excitée et éventuellement bruyante. Ma joie peut aussi être plus ou moins claire et évidente, explicite quant à ses objets et motivations.

Avec la joie comme acte de la conversion attentionnelle intéressée va de pair l'état d'excitation joyeuse (ou de torpeur) et la disposition gaie, de même que, dans le cas de la tristesse, l'état de torpeur, éventuellement <l'>état d'excitation du chagrin "passionné », la disposition basse, etc. Il s'agit là d'annexes de sentiments sensibles, qui forment éventuellement une unité intentionnelle avec l'acte originel, une unité du point de vue de l'aperception du sentiment : je suis tourné ou détourné avec passion.

[A VI 12 II/131 "53"] La difficulté consiste à tenir compte de la strate où <résident> les différences <entre> la joie [éprouvée avec une] "tranquillité d'âme », la joie "intempestive», passionnée, le bouleversement et la surprise de la joie (Freudenüberwältigung und -überraschung) - le cœur se tient tranquille et une grande vague de félicité afflue dans le cœur grand ouvert, puis affolement ou joie douloureuse, $<$ le $>$ cour menace d'exploser de joie $-<$ et $>$ l'amour uniforme, sans passion, et ainsi de suite. 
Nous parlions plus haut de l'être-intéressé, des degrés de l'immersion de l'ego dans le sentiment et du sentiment dans l'ego. Ne peut-on pas dire : dans la joie désirante et languissante (dans laquelle un désir languissant trouve son remplissement), il y a un rayon de l'orientation active de la joie qui part de l'ego vers l'objet et dans l'objet, plus précisément dans l'aimé, qui est en tant que tel un «bien», qui se tient là en tant que digne d'amour? L'amour enveloppe l'aimé, pour parler d'une manière imagée. Le collectionneur embrasse, pour ainsi dire, l'objet avec son sentiment, il s'adonne à lui, il va de l'intérieur vers l'objet en tant qu'il est doté de valeur. Les parties et les côtés de l'objet, les parties et les côtés axiologiques sont parcourus, et tandis que l'aperception et la position de valeur « saisit et pose » l'unité de la valeur, les composantes de valeur se "réalisent», et au plaisir de la réalisation et à son rythme est lié le rythme des sentiments dont consiste la jouissance (voir plus bas). Cette conversion attentionnelle dans la jouissance consiste dans des sentiments de remplissement, qui sont fondés à travers la réalisation des intentions axiologiques. Or, par rapport à cela, il y a aussi un contre-rayon. Au fait de se diriger vers l'objet et vers sa valeur correspond une direction opposée : celle qui retourne aux sentiments de la jouissance et à ce qui est rattaché à eux.

Je disais : C'est dans ces sentiments que consiste la jouissance. Cela n'est pas exact. Le flux des sentiments de joie proprement dits suscités par les intentions de valeur atteint la jouissance par la rétroconversion (durch die Rückwendung). [131 b] Le collectionneur jouit dans la mesure où il se réjouit en contemplant et en évaluant (il s'adonne donc dans sa joie à l'objet de valeur) et dans la mesure où, inversement, il s'adonne à la joie. Il n'éprouve pas seulement le vécu de la joie, le ravissement, ne vit pas seulement en lui sur le mode de l'orientation vers l'objet, mais il vit aussi dans le ravissement sur le mode de la jouissance, qui est jouissance de la joie, jouissance du ravissement. Il s'agit là d'une joie à deux niveaux: il n'est pas seulement heureux, mais se réjouit de son bonheur, et son état de bonheur s'enrichit de la sorte. De même pour la jouissance de l'amateur d'art. L'esthète a la convoitise de la joie esthétique, et dans la mesure où il a l'intuition de l'[objet] esthétique et s'en réjouit, son désir de cet objet se remplit à son tour dans la rétro-conversion, dans la jouissance de la joie esthétique.

Toutefois, il est manifeste que le deuxième niveau n'appartient pas à l'essence de chaque joie de la jouissance, ce qui entraînerait une régression à l'infini. C'est pourquoi nous pouvons mettre hors circuit ce deuxième niveau et dire : à la joie s'associe souvent une jouissance 
de la joie. Si la joie est très vive, elle s'impose à la conscience réflexive, à savoir à la conscience de valeur (au regard et à la joie à travers ce regard), et l'on a alors des tendances à la jouissance à propos d'une joie présente ou pareillement, de la tristesse à propos d'une tristesse présente (se sentir, s'estimer heureux - malheureux).

Si nous laissons donc de côté cette couche, deux choses demeurent :

1) À l'essence de la conscience de joie explicite et réalisée appartient une saisie de la valeur, un processus du déploiement réalisateur de valeurs, plus précisément de valeurs dont on se réjouit.

[A VI 12 II/132 a "54"] 2) La joie elle-même est le sentiment fondé ou motivé à travers la conscience qui saisit et perçoit les valeurs.

$<\S 6$. Différences et cohésion entre la conscience de valeur et le sentiment intentionnel de joie>

Maintenant se pose cependant la question du type et du sens de l'intentionnalité de cette joie proprement dite (et à ce dessein il faut noter que nous avons privilégié les cas de joie explicite, "claire et évidente »). N'est-il pas manifeste que l'intentionnalité de la joie ellemême est une autre que celle de la perception axiologique qui se trouve à son fondement?

On peut dire en effet: quelque chose se tient là pour moi en tant que pourvu de valeur dans la valuation, en tant que réjouissant dans la joie. Mais si nous regardons de plus près, la situation est pourtant essentiellement différente : le beau suscite l'appréciation du beau (la conscience de valeur) tout aussi peu <que $>$ la chose suscite la conscience représentative, ou tout aussi peu <que > le devoir-être pratique suscite la conscience du bien-être. Si des «états » sont suscités, nous nous sentons transposés dans des états, mais des «actes » ne peuvent pas être suscités. Des actes : à cela appartient l'aperception, les modes de la prise de position... La joie n'est pas une aperception (apperzipiert nicht). La joie n'a pas de modes de la prise de position et n'en est pas une. Elle est un état suscité à travers certaines aperceptions et prises de position. Par conséquent, le type d'intentionnalité consiste ici dans le fait que la valeur «apparaissante » ou consciente est le foyer de rayonnement d'une excitation, elle est source d'excitation pour quelque chose de suscité, pour l'état de l'ego.

On pourrait cependant objecter : La joie n'est pas une aperception, elle n'a pas un rôle constitutif pour des déterminités ontiques. Mais n'illumine-t-elle pas, ne laisse-t-elle pas la chose réjouissante appa- 
raître en tant que telle, à savoir dans une lumière rosée ? Et la tristesse n'assombrit-elle pas, ne laisse-t-elle pas la chose attristante apparaître en tant que telle, à savoir dans une lumière sombre et trouble? Le saint ne porte-t-il pas son nimbe, et la personne aimée son auréole, etc. ? Nous ne pouvons donc rien y faire, nous devons dire que la joie a elle aussi son "intentionnalité ", à savoir une certaine "relation à son objet». Et pourtant une tout autre que celle de l'attribution de valeur.

Nous pouvons exprimer la situation comme suit : la chose réjouissante possède un "éclairage » qui émane à partir de la joie comme à partir d'une source de lumière. La chose pourvue de valeur suscite de la joie, et la joie éclaire cette chose. Elle lui ajoute de la lumière, et en même temps elle est ce qui donne la lumière. Je peux examiner la lumière (l'éclairage, la lueur, l'éclat), je peux « voir » que la personne aimée est non seulement belle et estimable mais aussi " aimable». Elle a l'air aimable, etc. Mais de cette manière je me tiens aussitôt dans la relation entre ce qui est éclairé <illuminé> et ce qui éclaire. Je n'ai pas besoin de faire attention premièrement à la lumière qui éclaire, mais elle vient à la donation à travers cela, et je peux suivre le trajet de la lumière.

En quoi consiste l'orientation de l'amour, l'orientation, la joie à propos de l'objet ou à propos de son existence et de son être-tel ? Elle consiste dans le fait que la conversion attentionnelle qui attribue de la valeur suscite de la joie et un faisceau de rayons de cette joie se dirige sur les côtés et les parties de l'existence [de l'objet] concernés par l'attribution de valeur. Vivre dans la joie et, en elle, être tourné vers elle, cela veut dire que la joie est un vécu, mais non pas un objet, et que le fait de s'adonner à l'objet revient à s'adonner ${ }^{5}$ à sa valeur, quoique non pas à la valeur tout simplement, mais à celle qui est l'éclairage émanant de la joie. L'ego s'accomplit pour ainsi dire dans le rayonnement où se tient la configuration rayonnante, l'objet réjouissant et illuminé par la joie. Seulement, il n'y a pas [A VI 12 II/133 a "55"] d'acte de représentation proprement dit, pas d'acte $\mathrm{d}^{\prime}$ attention (se poster et se poser sur le mode de la représentation) qui se dirige vers la valeur, vers l'être-rosé, vers la lueur. La lumière de la joie rayonne sur l'objet de valeur en son entier. Mais les moments de valeur spécifiques ont leur relation particulière à la joie ou à la lumière comme "fondements », comme véritables foyers d'excitation de la joie ou points d'impact du rayonnement de la joie.

\footnotetext{
${ }^{5}$ Le terme « s'adonner » est employé dans les deux cas et donc douteux.
} 
Jusqu'à présent, nous avons considéré les actes de la conversion attentionnelle de la joie dans lesquels la conscience de valeur et la conscience de la joie se déploient de manière explicative : les cas de clarté et d'évidence dans la conscience de la joie. Dans les cas de confusion, l'objet représenté ou posé quant à son être est précisément représenté dans une représentation plus ou moins confuse ; sur cela se fonde une conscience de valeur confuse, qui n'est pas explicite ou claire. La joie suscitée est également confuse, sans une relation d'excitation déterminée à des composantes déterminées de l'attribution de valeur, et semblablement la lumière est un éclairage général, sans des fondations axiologiques clairement détachées.

L'image de l'éclairage n'est pourtant pas adéquate à tout point de vue, malgré la force avec laquelle elle s'impose. Ce qui fait défaut dans cette image, c'est un analogon pour la relation particulière qu'entretient le faisceau de rayons éclairants avec les côtés clairement constitués de la valeur, ou pour la manière particulière dont les caractères d'éclairage appartiennent à certains côtés de cette sorte, tandis que cette relation fait défaut pour d'autres côtés. L'objet est agréable, aimable, réjouissant grâce à tel ou tel moment ; ceux-ci sont ce qui est, dans l'objet, réjouissant à proprement parler, alors que tout - 1'objet en son entier - est inondé de lumière. Là où aucune particularisation et aucune élucidation n'a lieu, là où l'objet est rendu conscient par une représentation et par une position impropre et vide, c'est [133 b] un éclat général qui va dans cette direction intentionnelle, éclat qui peut néanmoins être très vif, même si les différences de vivacité de la joie relèvent d'un autre plan. D'habitude, une lumière particulièrement vive, donc particulièrement puissante rend clair, mais cette lumière de la joie ne rend pas clair, et si elle a une évidence à elle, c'est grâce à d'autres sources.

Allons encore plus loin! Prenons le cas suivant: j'ai parlé à la personne aimée et j'ai été rempli de joie à son égard. Je peux m'être entretenu avec elle à propos de choses indifférentes qui ont été le thème [de notre conversation]. Les actes de perception de la valeur n'ont pas été thématiques, je n'ai pas vécu dans la saisie de la valeur et non plus dans la conversion attentionnelle thématique de la joie en rapport avec sa beauté, sa bonté, sa gentillesse, etc. Et pourtant cela ne manque pas à une conscience de ces propriétés axiologiques et à une joie peut-être vivace, cela ne manque pas non plus à l'éclaircissement qui, partant de la joie, embrasse l'être de la personne aimée et plus précisément son être dans l'apparition. Il n'y a donc ici aucune conversion attentionnelle thématique de la joie ; l'attribution de valeur et la joie se rapportent, chacune à sa façon, à l'objet, mais la 
conversion attentionnelle proprement dite, la conversion attentionnelle de la visée (meinende Zuwendung) fait défaut. (La personne aimée elle-même n'est pas le thème. Et pourtant, tout ce qui se tient dans une relation à elle s'imposera plus aisément à moi, et lorsque c'est le cas, ce sera d'une manière centrée sur elle). Cet être-centré est apparenté à l'être-thématique, comme si la personne aimée était un thème caché : et pourtant, la conversion attentionnelle de la visée fait défaut.)

Il faut encore discuter la différence entre les cas où la joie s'édifie à partir d'une représentation, position, perception axiologique actuelle, et les cas où elle se fonde sur la rétention. La personne aimée a quitté la pièce. Je reste cependant tourné vers elle. [A VI 12 II/134 a "56"] Des images d'elle dans le souvenir, des représentations vides et des positions sont des éléments intermédiaires pour des conversions attentionnelles de joie et d'attribution de valeur. Par exemple, le beau est subitement voilé, il n'est plus visible, mais je reste dirigé sur lui. Il se peut pourtant aussi que je continue de vivre dans le sentiment de bonheur, de ressentir la joie heureuse qui me remplissait à l'égard de l'existence pleine de vie et de la manière dont la personne aimée se donne sans que je dirige un regard qui les vise vers elles et vers leur éclaircissement. Il se passe alors quelque chose de cette sorte : je suis tourné à présent vers la félicité elle-même et je jouis du bonheur, dans un sentiment de joie de deuxième niveau (ce cas a déjà été mentionné). Dans ce cas comme dans le cas précédent, une certaine relation à l'objet qui est source de bonheur est encore vivante, une conscience représentative et qui attribue de la valeur est encore présente. (Même si dans le dernier cas le rayon de la visée s'en est retiré ; l'intention spécifique ne suit pas la lumière de la direction de rayonnement de la joie, et la lueur rosée, la lumière qui émane de la joie, devient confuse.)

Il y a bien sûr aussi des différences quant au fait de savoir si les moments donateurs de valeur sont eux-mêmes donnés en personne selon qu'est accomplie une intuition de l'objet conformément à ses propriétés fondatrices de valeur, ou s'il n'est question de l'objet que de manière discursive: j'entends une nouvelle réjouissante, la lumière de la joie inonde la proposition, l'énoncé en tant que tel et aussi, ce faisant, l'état de choses; il se peut aussi qu'il y ait une accentuation eu égard aux mots particuliers et aux choses particulières qui posent la valeur. Mais cela a besoin de clarté. Les fondements de la valuation ne sont pas donnés, l'état axiologique est posé seulement selon la pensée, et la valeur elle-même est posée simplement dans la pensée. 
[134 b] Encore un cas nouveau : La personne aimée est sortie de la pièce, je suis rempli de bonheur, mais sans être tourné vers son "image » (rétention), et sans être non plus tourné vers la félicité ou le bonheur. Je prends un livre dans la main, je regarde autour de la pièce : tout a reçu de sa présence attrait et valeur. Je me maintiens ici dans l'unité thématique, et les actes impliquent encore des relations et des visées (fussent-elles secondaires) qui vont vers la personne aimée. Je me réjouis de toutes ces choses, mais en vertu de la relation à la personne aimée. Sa présence a consacré cet espace, son commerce avec ces choses leur a donné de la valeur, etc. Il n'est besoin en ce sens de diriger aucun rayon de visée propre sur la personne aimée, dans le souvenir. Nous sommes dirigés vers les choses, vers leurs caractères de valeur et vers leur éclairage par la joie. Mais ces joies ont une cohésion avec la félicité vivante qui me remplit, rayonnent à partir d'elle, quoique d'une manière autre que lorsqu'elles étaient l'objet de la félicité. Et à plus forte raison, lorsque <je> dirige mon regard vers le reste du monde ambiant: je me réjouis de la lumière qui est posée sur tout. Comme le monde est beau en tout! Le monde en son entier reçoit de la lumière à partir de la personne aimée, etc. Dans l'ivresse de la félicité, j'en suis entièrement «rempli ». Je me tourne tantôt vers l'objet qui fait ma félicité, tantôt vers la félicité [elle-même], vers les objets qu'elle s'approprie dans la joie et qui lui étaient avant extérieurs, en jouissant de leurs reflets. Dans tout cela, la joie, l'état de joie, la félicité conserve sa direction intentionnelle au sens plus large, même si son être-dirigé sur le mode de la visée fait défaut. [A VI 12 II/135 a "57"] Maintenant, est-ce que la joie suscitée à propos d'A peut perdre la relation à l'objet, la relation à la valeur d'A qui l'a suscitée?

Quelque chose me donne de la joie. Je suis alors gai, bien disposé. Dans la disposition gaie je ne me réjouis plus à propos d'A; je sais pourtant encore que je suis gai parce qu'A m'a rendu gai. Il se peut cependant aussi que, consécutivement, plusieurs choses réjouissantes indépendantes l'une de l'autre m'aient rendu gai. Quant à la question du motif de la gaieté, il se peut que je ne me souvienne plus de l'une ou de l'autre, que je ne puisse désigner toutes les raisons, et peut-être même que je ne puisse indiquer aucune raison déterminée.

Une telle gaieté, en tant que disposition, peut bien avoir été originellement joie à propos d'A ou d'A', elle ne l'est plus, elle est privée de la relation déterminée à ses raisons originelles de stimulation. Il n'est pas non plus besoin qu'elle soit précédée par un grand choc de l'excitation: un courant et un habitus de disposition de vie joviale peut aussi être provoqué par de petites stimulations renouvelées. 
Des sentiments de joie et des dispositions joviales peuvent aussi surgir sans l'arrière-fond d'une intentionnalité thématique qui attribue de la valeur, autrement dit, sans s'établir comme des joies qui se dirigent spécifiquement vers une chose de valeur ; ainsi, par exemple, dans le cas de la joie de la recherche scientifique, ou plutôt en tant qu'elle est une joie eu égard au rythme des positions et des résolutions de problèmes à travers de nombreuses déceptions, de nombreux obstacles et les désagréments correspondants, cas dans lequel l'intention thématique réside sûrement dans la conscience théorique. Chaque joie de cette sorte a sa « relation » à ce qui la fonde et la suscite, sans qu'il y ait là une orientation thématique. Semblablement, le rythme de la vie dans ses couches inférieures avec ses représentations, ses jugements, ses attributions de valeur et ses aspirations peut comporter une couche plus profonde (ou plus élevée) du sentiment dans le rythme, mais sans orientation. [135 b] Même des pensées émergeant en cachette (des souvenirs, des représentations de toutes sortes), qui ne sont traversées par aucun rayon de la visée, peuvent fonder, susciter des sentiments, joviaux ou tristes, plaisants $<$ ou> déplaisants, qui peuvent couler dans un flux unitaire du sentiment, dans une unité de disposition.

$C^{\prime}$ est à cela qu'appartient aussi le grand flux des sentiments sensibles, des sentiments communs, et à ce propos il faut aussi dire que de chaque joie vive partent des flux de sentiments sensibles qui accroissent et élargissent l'excitation de la joie, et même que, comme cela apparaît, chaque excitation de la joie a ses composantes sensibles et une ample teneur de plaisir sensible, qui toutefois a un côté spirituel et éventuellement une orientation vers le spirituel, à travers le rapport d'excitation à l'éventuelle origine fortement spirituelle, à travers son origine dans des sphères esthétiques, scientifiques, religieuses. 